\title{
Road Map and Challenges in 4G Wireless System
}

\author{
A. K. Rathore*, R. K. Chaurasia, R. Mishra and H. Kumar \\ Department of Electrical and Computer Engineering, National University of Singapore, Singapore
}

\begin{abstract}
The user demand for high speed multi-media application has been increasing many folds in the recent years. New smart phones are launching in the market. The wireless communication is looking forward for a new generation technology that will match the application demand and resources. This is where the fourth generation (4G) wireless communication is required. $4 \mathrm{G}$ wireless communication is expected to provide better speed, high capacity, low cost IP based services. The main objective of the $4 \mathrm{G}$ wireless communication is to supersede the current core technology with a single unified technology. More and more advanced interactive multimedia applications are emerging as $4 \mathrm{G}$ networks resulting in a rapid development of video coding and communication techniques. This paper deals with the current scenario and the future prospects of the $4 \mathrm{G}$ system in addition with the challenges it is going to witness.
\end{abstract}

Keywords: Broadband wireless access; Content delivery; Subcarrier; Multimedia services

\section{Introduction}

$4 \mathrm{G}$ or Fourth generation is the future technology for mobile and wireless communications. Approximately $4 \mathrm{G}$ deployments are expected to be around the year 2010 to 2015. It will be the successor of $3 \mathrm{G}$ network technology. Voice is the basic drive force for the second generation $(2 \mathrm{G})$ mobile communication [1], whereas video and television services are the chief factor for $3 \mathrm{G}$. In the case of $4 \mathrm{G}$ low cost and high speed data are the dominant driver. High degree of personalization, application ubiquity and synchronization between user appliances are also the driven forces. The evolution to $4 \mathrm{G}$ from $3 \mathrm{G}$ will be driven by the services that offer better quality in multimedia, voice and sound. Personalization will be improved with greater bandwidth and sophistication in large quantity of information. Convergence with other network service will come through high session data rate. The impact on the network capacity is expected to be significant.

Video communication is facing many challenges now. $4 \mathrm{G}$ wireless networks will provide many features to handle this [2]. It will provide the end-users more opportunities and flexibilities in accessing video and multimedia contents. It accommodates radio access systems via flexible core IP network, resulting in a very high data rate, and enhancing the video applications. It provides better quality of service and security supports to the end users, and enhances the user experiences and privacy for multimedia applications. The quest for video applications and services would bring in a large volume of data transmission onto the wireless network. This is going to be a potential challenge for the $4 \mathrm{G}$ systems.

Now a day it is quite interesting to note that the new mobile phones, specially the smart phones, are not just the simple phone but much more than that. They are a little mobile PC and functions more or less like that. They includes a keyboard rendered on a touch screen, provide user friendly graphical user interfaces, provide internet services, web browsing, email connecting, local wireless fidelity connectivity, built in camera, high quality music player capability, and small media management besides phone call functionalities.

However there are many features that some smart mobile do not support yet. This includes mobile television support to receive live programmes, multi user networked three- dimension games, realistic $3 \mathrm{D}$ scene rendering and high definition visuals. The lack of these functions are due to computational capabilities and power constraints of the mobile devices, available bandwidth and transmission efficiency of the wireless network, universal access capability of the infrastructure, and the compression and error control efficiency of video and graphics data. Mobile phones are moving faster to provide the user the same or even better experience than the personal computer, but there is long way ahead. From a mobile communication point of view, it is expected to have a higher data transmission rate as comparable to wire line network. Also the expectation is over service and support for seamless connectivity and access to any application regardless of device and location. The expectation of mobile communication is the key feature of $4 \mathrm{G}$. It is going to describe the next complete evolution in wireless communication. It is also expected to provide a comprehensive IP solution where multimedia services can be delivered to the user on an 'anywhere anytime' basis with a satisfactory data rate and high security. The current $3 \mathrm{G}$ wireless infrastructure is not able to achieve this demand. The International Telecommunication Union (ITU) is working on the standard and targets for the commercial deployment of $4 \mathrm{G}$ system in the year 2010-2015 timeframe. ITU defined IMTadvanced as the successor of IMT-2000 or 3G $[3,4]$.

The advantage of $4 \mathrm{G}$ over $3 \mathrm{G}$ is listed in table 1 below. It is obvious that $4 \mathrm{G}$ has much more system improvement over $3 \mathrm{G}$. This improvement is not only in bandwidth and capacity, but it also appears in coverage, latency, mobility and security.

\section{Status \& Key Technologies}

In order to discuss the multimedia communications across $4 \mathrm{G}$ networks, it is obvious to unfold the key technologies and innovations in $4 \mathrm{G}$. The $4 \mathrm{G}$ system will include all the standards that the earlier generations have been using. The figure 1 shows the evolution in the technologies from $1 \mathrm{G}, 2 \mathrm{G}$, and $3 \mathrm{G}$ to $4 \mathrm{G}$ in wireless systems. $4 \mathrm{G}$ also contain the wireless local area network system to broadband wireless

*Corresponding author: Akshay K Rathore, Department of Electrical and Computer Engineering, National University of Singapore, Singapore, E-mail: eleakr@nus.edu.sg

Received June 17, 2012; Accepted July 18, 2012; Published July 21, 2012

Citation: Rathore AK, Chaurasia RK, Mishra R, Kumar H (2012) Road Map and Challenges in 4G Wireless System. J Elec Electron 1:104. doi:10.4172/2167101X.1000104

Copyright: (c) 2012 Rathore AK, et al. This is an open-access article distributed under the terms of the Creative Commons Attribution License, which permits unrestricted use, distribution, and reproduction in any medium, provided the original author and source are credited. 


\begin{tabular}{|c|c|c|}
\hline & $3 G$ & $4 G$ \\
\hline Architecture & WAN & W-LAN \& WAN \\
\hline Information type & Voice is prime, data secondary & $\begin{array}{l}\text { Converged data and } \\
\text { multimedia services }\end{array}$ \\
\hline Data rate & $384 \mathrm{kbps}-2 \mathrm{Mbps}$ & $\begin{array}{l}100 \text { Mbps (moving), } \\
1 \text { Gbps (standing) }\end{array}$ \\
\hline Frequency & $18-2.4 \mathrm{GHz}$ & $2-8 \mathrm{GHz}$ \\
\hline Class of switching & Both circuit and packet & Only packet \\
\hline Multiple access technology & CDMA & OFDMA \\
\hline Broadcast & Not supported & Supported \\
\hline Quality of service & Less supported & Supported \\
\hline
\end{tabular}

Table 1: Comparison of $3 G$ and $4 G$ wireless system.

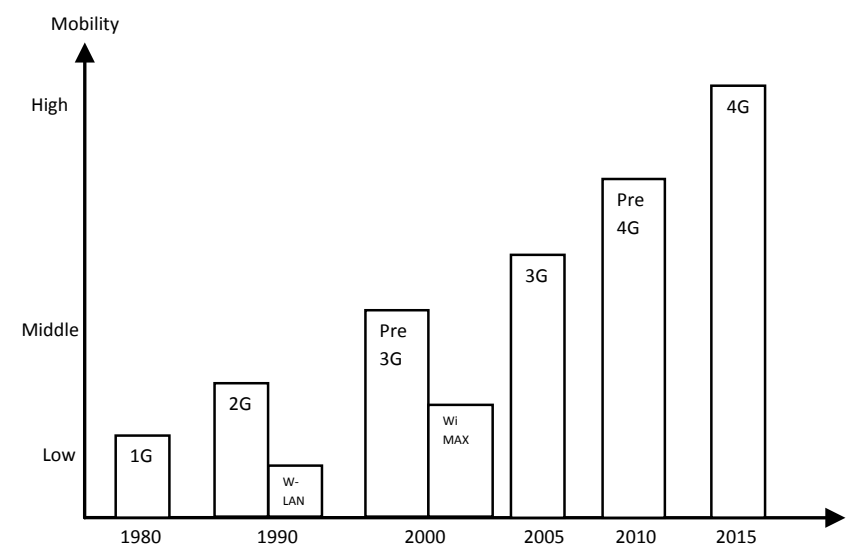

Figure 1: Evolving of technologies and their mobility.

access. 3GPP LTE/ LTE - advanced, 3GPP2 UMB and Mobile WiMAX based on IEEE $802.16 \mathrm{~m}$ are the technologies that are widely adopted for the deployment of $4 \mathrm{G}[5]$.

One of the unique features of $4 \mathrm{G}$ networks is that they will accommodate heterogeneous radio access systems, which will be connected via flexible core networks. Thus, an individual user can be connected via a variety of different access systems to the desired networks and services. The interworking between these different access systems in terms of horizontal and vertical handover and seamless service provision with service negotiation including mobility, security and quality of service management will be a key requirement. It may be handled in the core network or by suitable servers accessed via the core network. This optimally connected anywhere, anytime view could be realized by a network comprising a variety of interworking access systems connected to a common packet-based core network.

IMT-Advanced has been developed to provide true end-to-end IP services to mobile users at anytime and anywhere basis. Although the standardization process is still ongoing, the major design goals of $4 \mathrm{G}$ are quite certain. It includes that $4 \mathrm{G}$ will be all IP networks. This means that circuit switching will be eliminated in the next-generation cellular networks. Another point is that $4 \mathrm{G}$ will have a very high data rate. It is expected that $4 \mathrm{G}$ networks will be capable of providing $100 \mathrm{Mbps}$ data rate under high mobility, which is much faster than $3 \mathrm{G}$. Next point is that $4 \mathrm{G}$ will provide quality of service and security to the end users. This feature has been lacking in 3G networks. And the last one is that IP-based multimedia services such as Voice over IP (VoIP) and video streaming are expected to be the major traffic types in $4 \mathrm{G}$ networks $[6,7]$.

Long Term Evolution (LTE) was introduced in 3GPP (3
Generation Partnership Project) as the major step for Universal Mobile Telecommunication System (UMTS) [8]. Its function is to provide an enhanced user experience for broadband wireless networks. It supports a bandwidth ranging from $1.25 \mathrm{MHz}$ to $20 \mathrm{MHz}$. It supports both frequency division duplex and time division duplex. The peak downlink rate is $100 \mathrm{Mbps}$ and peak uplink rate is $50 \mathrm{Mbps}$ in a $20 \mathrm{MHz}$ channel. Its high speed is achievable because of great improvement in the spectral efficiency. It has a low latency of the order of $100 \mathrm{~ms}$ for and $5 \mathrm{~ms}$ for control-plane and user-plane respectively. LTE also supports a seamless connection to all the existing networks such as GSM, CDMA and HSPA. LTE also provides IP based traffic as well as quality of service for the multimedia services.

LTE uses a multiple input multiple output (MIMO) system in order to achieve high throughput and spectral efficiency. It uses $2 \times 2$ (two transmit antennae at the base station and two receive antennae at the terminal side), $3 \times 2$ or $4 \times 2$ configuration for downlink [9]. In addition, LTE supports multimedia broadcast multicast services (MBMS) either in single cell or multi-cell mode. Most carrier supporting the GSM or HSPA networks have upgraded their system to LTE. Carriers using different standards are also upgrading their system to LTE.

UMTS terrestrial radio access evolving from LTE has two different air-interface technologies. Here the downlink is based on OFDMA and the uplink is based on SC-FDMA.

Conventional OFDM is widely a key technology for the downlink transmission scheme for E- UTRA frequency division duplex and time division duplex modes [10]. Here the available spectrum is divided into multiple sub-carriers, and each sub-carrier is modulated independently by a low rate data stream as compared to OFDM. This orthogonal OFDM allows for the multiple users to access the available bandwidth. It assigns specific time-frequency resource to each individual. According to scheduling here the data channels are shared by multiple users. The complexity of OFDMA is increased but this result in efficiency and latency.

OFDMA signal has weaker peak to average power, therefore it results in worse uplink coverage. So the uplink transmission is based on Single Carrier FDMA. The signal processing for both these technologies are same, hence the parameterization of downlink and uplink can be harmonized. Here DFT- spread- OFDM has been selected for E-UTRA, where a size M DFT is applied to a block of M modulation symbols, and then DFT transforms the modulation symbols into the frequency domain [5]. The resultant signal is then mapped onto the available subcarriers. DFT processing is the fundamental difference between SCFDMA and OFDMA signal generation. Each subcarrier of an OFDMA signal only carries information related to a specific modulation scheme, whereas SC-FDMA contains all the transmitted modulation symbols because of the spread process.

The main advantages of Orthogonal Frequency Division Multiplexing (OFDM) is that it supports a high data rate by dividing an entire channel into many overlapping narrow-band sub-channels by using orthogonal signals [11]. Different subcarriers can be allocated to different users in order to provide a flexible multiuser access scheme and to exploit multiuser diversity. It also deals efficiently with frequencyselective fading without the need for a complicated equalizer. OFDM has a high degree of flexibility of radio resource management. It provides different frequency responses of different channels for various users, data rate adaptation over each subcarrier, dynamic sub-carrier assignment and adaptive power allocation.

OFDM suffers in some aspects also. One of the major drawbacks 


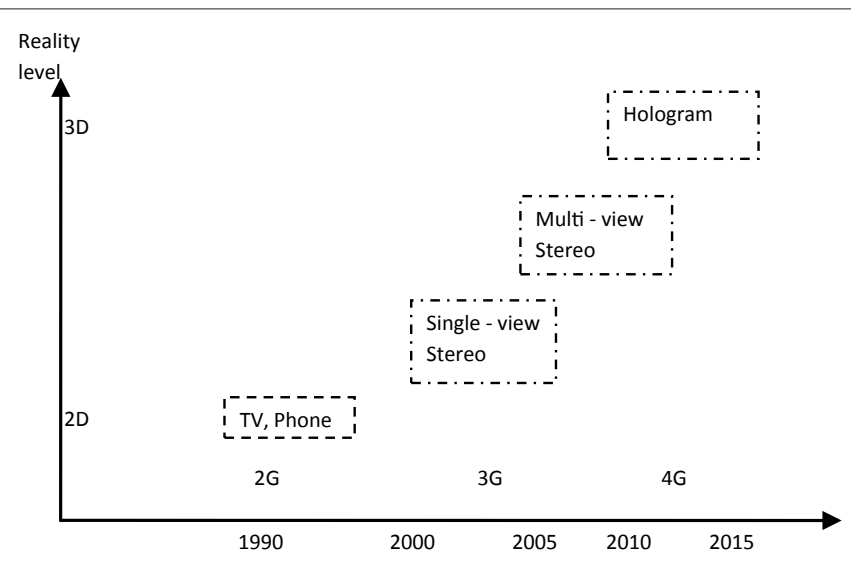

Figure 2: Estimated reality video over wireless networks.

of it is that signals have a large peak to average power ratio. This ratio increases approximately linearly with the number of subcarriers. Large peak to average power ratio force the transmitter power amplifier to have a large backoff in order to ensure the linear amplification of the signal. The high peak to average power ratio of OFDM is reduced by clipping the OFDM signal above some threshold, peak cancellation with a complementary signal, allowing nonlinear distortion from the power amplifier and special coding techniques. Also OFDM systems are highly sensitive to frequency offsets caused by the oscillator inaccuracies and the Doppler shift due to mobility, which gives rise to inter-carrier interference. Methods of reducing frequency offset effects include windowing of the transmitted signal, self interference cancellation schemes and frequency offset estimation methods.

\section{Challenges and Opportunities}

It may be expected that in $4 \mathrm{G}$ the communication networks will continue to expand. It will include all kinds of channels with various information, quality of service and protocols. It will also contain all heterogeneous terminals with a diverse range of capabilities, accessibilities and user preferences. The world today is moving to a content producer. Uploading one's piece of work onto the network and enjoying the work of other is the trend. The gap between the richness of multimedia content and the variation of techniques for content access and delivery will increase dramatically. Expectation of universal multimedia access (UMA) will be a challenge for the $4 \mathrm{G}$ wireless networks [12]. The major concept of UMA is universal or seamless access to multimedia content by automatic selection or adaption of content.

Content analysis refers to intelligent computational techniques. It extracts information automatically from a recorded video sequence/ image. From such analysis, the machine is able to discover what is presented in the scene, who is there and where or when it occurs. As people have become more and more enthusiastic about watching multimedia content using mobile devices, so they personalize the content by summarizing the video for easy retrieval or for easy transmission. Salient images extracted automatically from the captured video sequence by a mobile phone camera. This is referred as video summary. It is to be delivered to remote friends so as to share the exciting experience of watching a sport match. The summarizer identifies the exciting moments of the video based on certain analysis criteria so that the content coverage is guaranteed while a great deal of bandwidth and battery power for transmitting the entire video are saved. Here it plays a significance role in $4 \mathrm{G}$ wireless communications.

Content based interactivity is highly connected to UMA. It also imposes higher expectations and requirements on content understanding supports. In the $4 \mathrm{G}$ wireless system, the mobile television user may customize the content by manipulating the text, image, audio, video and graphics. As this system is high speed system so the user may even access information that is not available and supported in the current system. The $4 \mathrm{G}$ wireless system is going to face a challenge for the watching movie or television in a new user experience with much higher interactivity. Also video on TV will not be flat for much longer time. The next step forward is three dimension (3D) video / TV services over $4 \mathrm{G}$ wireless networks with various representations [13]. The trend is shown in figure 2 below. At present the stereophonic and multi-view $3 \mathrm{D}$ videos are more developed than other $3 \mathrm{D}$ video representation formats owing to the standardized MPEG in coding approaches. Normally $3 \mathrm{D}$ video takes 1.5 times bit rate as required by $2 \mathrm{D}$ video, but using efficient coding, such as sub-band based coding, the coded 3D video takes a 1.2 bit rate as compared to traditional $2 \mathrm{D}$ video [14-16]. Therefore higher reality requirements will bring a larger volume of data to be delivered over the network. This in addition to more services and user scenarios are the challenges for $4 \mathrm{G}$ wireless systems.

Cross-layer design mechanism plays a critical role in order to supply so many applications and services with quality of service in $4 \mathrm{G}$ networks. In the cross layer design the interactions between the different network protocol layers can be optimized jointly in end-toend system. This is done in order to achieve better performance. The content delivery with satisfactory quality of user experience becomes an important issue.

\section{Conclusion}

Currently 4G wireless communication systems are still in their planning phase. The new infrastructure is expected to provide much higher data rates, lower cost per transmitted bit, more flexible mobile terminals, and seamless connections to different networks. In this paper, we introduced the features of $4 \mathrm{G}$, and also point out the short coming of the existing technology. $4 \mathrm{G}$ seems to be the interesting technology which the world is looking forward. It is also a cardinal one to the emerging mobile phones, and has many unique features. High speed multimedia based services are its essential features. $4 \mathrm{G}$ wireless networks provide many features to handle the current challenges in video communications. Content delivery will play a major role here. A demand for a larger volume of data to be delivered over the network is the priority. These services entangled with user scenarios are the challenges for $4 \mathrm{G}$ wireless systems.

\section{References}

1. Rappaport TS (1996) Wireless Communications: Principles and Practice. Prentice Hall PIR.

2. Chia JB (2002) Video services over 4G wireless networks: not necessarily Streaming. Wireless Communications and Networking Conference, 2002. WCNC2002. 2002 IEEE 1: 18 - 22.

3. Bria A, Gessler F, Queseth O, Stridh R, Unbehaun M, et al. (2001) 4thgeneration wireless infrastructures: scenarios and research challenges Personal Communications, IEEE 8: 25-31.

4. Sun JZ, Sauvola J, Howie D (2001) Features in future: 4G visions from a technical perspective. Global Telecommunications Conference, 2001. GLOBECOM'01, IEEE 6: 3533 - 3537 
Citation: Rathore AK, Chaurasia RK, Mishra R, Kumar H (2012) Road Map and Challenges in 4G Wireless System. J Elec Electron 1:104. doi:10.4172/2167-101X.1000104

Page 4 of 4

5. 3GPP TD RP-040461: Proposed Study Item on Evolved UTRA and UTRAN

6. Hui SY, Yeung KH (2003) Challenges in the migration to $4 \mathrm{G}$ mobile systems, Communications Magazine, IEEE, 41: 54-59.

7. Wang J (2001) Broadband Wireless Communications: 3G, 4G and Wireless LAN. Academic Publishers.

8. (2007) C.S0084-000-0, Overview for Ultra Mobile Broadband (UMB) Air Interface Specification.

9. Biglieri E, Calderbank R, Constantinides A, Goldsmith A, Paulraj A, et al. (2007) MIMO Wireless Communications. Cambridge University Press.

10. Keller T, Hanzo L (2000) Adaptive multicarrier modulation: a convenien framework for time-frequency processing in wireless communications. Proceedings of the IEEE, 88: 611-640.

11. Bingham JAC (1990) Multicarrier Modulation for Data Transmission: An Idea Whose Time Has Come, IEEE Communications Magazine 28: 5-14.
12. Stamou G, Kollias S (2005) Multimedia Content and the Semantic Web. John Wiley \& Sons.

13. Dane G, El-Maleh K, Wang H (2007) A multi-mode video object segmentation scheme for wireless video applications. Computer Communications and Networks, ICCCN , Honolulu, USA

14. Sayood K (1996) Introduction to Data Compression. Morgan Kaufmann Publishers.

15. Wang H, Schuster GM, Katsaggelos AK (2005) Rate-distortion optimal bit allocation scheme for object-based video coding. IEEE Trans. Circuits and System for Video Technology.

16. Pan F, Lin ZP, Lin X, Rahardja S, Juwono W, et al. (2003) Content adaptive frame skipping for low bit rate video coding, in Proc. 2003 Fourth International Conference on Information, Communications and Signal Processing 1: 230234 